\title{
Left Ventricular Hypertrophy with Strain by ECG Finding
}

National Cancer Institute

\section{Source}

National Cancer Institute. Left Ventricular Hypertrophy with Strain by ECG Finding. NCI

Thesaurus. Code C102655.

An electrocardiographic finding suggestive of a hypertrophied left ventricle, characterized by large QRS amplitudes, ST depression and T wave inversion. (CDISC) 